\title{
LES ENGAGEMENTS PAR PAYS : INTENDED NATIONALLY DETERMINED CONTRIBUTION (INDC)
}

Sont donnés ici quelques exemples commentés d'engagement déposés par un certain nombre de pays, à l'automne 2015, avant la tenue de la conférence de Paris.

Nota 1 : les objectifs affichés par les pays ne sont pas tous formulés dans les mêmes unités : selon qu'ils sont évalués en tonne de $\mathrm{CO}_{2}$ par habitant ou par unité de PIB, selon les hypothèses implicites de croissance de la population ou du PIB sur les 20 prochaines années, les quantités totales émises par le pays peuvent, à terme, être très différentes.

Nota 2 : les seules statistiques détaillées par pays, qui soient disponibles, sont anciennes (année 2011) et ne concernent que les émissions de $\mathrm{CO}_{2}$, pas celles d'autres gaz à effet de serre, avec leur coefficient d'équivalence. 


\section{LES ÉTATS-UNIS}

Population : 314,9 millions; Production $\mathrm{CO}_{2}: 16,85 \mathrm{t} / \mathrm{h}$ (11 $\left.{ }^{\mathrm{e}} \mathrm{rang}\right)$; PNB : $49277 \$ / h^{6}$

Les États-Unis s'engagent dans leur INDC à réduire leurs émissions par rapport à 2005 de $17 \%$ en 2020 et de 26 à $28 \%$ en 2025 . Il faut noter que l'objectif pour 2020 consiste à revenir un peu au-dessus des émissions de 1990-1991. Pour parvenir à ce résultat, les États-Unis citent une série de lois et réglementation existantes ou en cours, mais sans précision sur les moyens.

\section{LA CHINE}

\section{Population : 1368,4 millions; Production $\mathrm{CO}_{2}: 6,59$ t/h (44 ${ }^{\mathrm{e}}$ rang);} PNB : $10092 \$ / h$

Les engagements de la Chine consistent à réduire les émissions de $\mathrm{CO}_{2}$ de 40-45\% par unité de $\mathrm{PIB}$ en 2020 par rapport à 2005. Sachant que ces émissions auraient été réduites de 33,8 \% en 2014 par rapport à 2005 , ceci correspond à un effort de réduction supplémentaire de 18 à $33 \%$ par unité de PIB en 2020 par rapport à 2014. Compte tenu de la croissance probable du PIB, ceci devrait correspondre en pratique à une augmentation des émissions significative. Dans ce cadre, la Chine prévoit d'augmenter la part des combustibles non fossiles dans la consommation d'énergie primaire de 11,2 \% en 2014 à $15 \%$ en 2020 et d'augmenter les surfaces forestières d'une vingtaine de millions d'hectares. Par ailleurs, la Chine prévoit atteindre le pic de ses émissions totales de $\mathrm{CO}_{2}$ en 2030.

6. Source: Carbon Dioxide Information Analysis Center (CDIAC), US Department of energy (DOE), données 2011. 


\section{L'INDE}

\section{Population : 1 221,1 millions; Production $\mathrm{CO}_{2}: 1,69 \mathrm{t} / \mathrm{h}\left(114^{\mathrm{e}} \mathrm{rang}\right)$; PNB : $4786 \$ / h$}

Comme la Chine, l'Inde prévoit de réduire ses émissions par unité de PIB, mais seulement de 33-35\% en 2030 par rapport à 2005, ce qui devrait correspondre à une forte augmentation des émissions totales. Dans ce cadre, l'Inde prévoit de porter la capacité de production d'électricité hors combustibles fossiles à $40 \%$ de la capacité installée et de créer 2,5 à 3 milliards de tonnes de puits d'équivalent $\mathrm{CO}_{2}$ par un accroissement de la couverture forestière.

\section{L'UNION EUROPÉENNE}

Population : 508,7 millions; Production $\mathrm{CO}_{2}: 7,04$ t/h (40 $\mathrm{rang}$ ); PNB : $34364 \$ / h$

L'Union européenne s'engage à une réduction de $40 \%$ de ses émissions de gaz à effet de serre en 2030 par rapport à 1990 en rappelant ses engagements antérieurs de réduire ses émissions de $20 \%$ entre 1990 et 2020 et le fait que ces émissions ont déjà été réduites de $19 \%$ dès 2012. Les émissions sont donc passées de 12 tonnes d'équivalent $\mathrm{CO}_{2}$ par habitant à 9 tonnes en 2012, en visant 6 tonnes en 2030.

\section{L'ÉGYPTE}

Population : 79,4 millions; Production $\mathrm{CO}_{2}: 2,78 \mathrm{t} / \mathrm{h}$ (94 $\mathrm{rang}$ ); PNB : $10631 \$ / \mathrm{h}$

Le document remis par l'Égypte rappelle les circonstances particulières dans lesquelles le pays se trouve placé : croissance attendue de la population (croissance annuelle supérieure à $2 \%$ jusqu'en 2040) et du PIB, avec un accent mis sur de grands projets (+ $5 \%$ par an); amélioration des indicateurs sociaux. Il insiste sur les effets négatifs attendus du changement climatique : diminution des rendements 
agricoles (de près de $20 \%$ pour les céréales, d'ici 2050); submersion des zones littorales du delta...

Il donne ensuite des indications assez exhaustives, mais très générales, sur les moyens de réduire les émissions de GES, sans aucun objectif chiffré. Il évalue, enfin, les investissements à réaliser sur la période 2020-2030 à $73 \mathrm{MM} \$$, pour lesquels le support international est attendu.

\section{L'AFRIQUE DU SUD}

Population : 52 millions; Production $\mathrm{CO}_{2}: 9,19 \mathrm{t} / \mathrm{h}\left(27^{\mathrm{e}}\right.$ rang); PNB : 12197 \$/h

L'Afrique du Sud présente des outils de planification qui intègrent élimination de la pauvreté, éradication des inégalités et lutte contre le changement climatique. L'objectif affiché est un niveau d'émissions de GES en 2025 et 2030 entre 398 et 614 Mt d'équivalent $\mathrm{CO}_{2}$, à comparer à un niveau actuel de $477 \mathrm{Mt}$; l'évolution prévue sur la période comporte d'abord une croissance des émissions puis un plateau et enfin une décroissance. Sont également données des estimations des investissements qui sont prévus, à la fois pour réduire les émissions et lutter contre les conséquences du changement climatique.

\section{L'ÉTHIOPIE}

Population : 89,4 millions; Production $\mathrm{CO}_{2}: 0,084 \mathrm{t} / \mathrm{h}\left(184^{\mathrm{e}} \mathrm{rang}\right.$ mondial); PNB : 1172 \$/h

L'Éthiopie affiche un objectif de niveau d'émission de GES, en 2030, identique, en niveau absolu, par rapport à ce qu'il est en 2010, ce qui représente un effort de réduction de $64 \%$ par rapport à la prévision de croissance de sa population et de développement de son économie. Elle prévoit également de devenir exportatrice d'électricité «verte», d'origine hydraulique, dans sa région. 